\title{
Home Treatment of Presumed Melanocytic Nevus With Frankincense
}

\author{
Davida Kornreich, MD; Nazanin Saedi, MD
}

\section{PRACTICE POINTS}

- Many patients seek natural methods of home mole removal online, including topical application of essential oils such as frankincense.

- These agents often are unregulated and can be potentially harmful when used without appropriate supervision.

- Dermatologists should be aware of common online fallacies to better answer patient questions and guide them to more accurate sources of dermatologic information and appropriate treatment.

To the Editor:

Melanocytic nevi are ubiquitous, and although they are benign, patients often desire to have them removed. We report a patient who presented to our clinic after attempting home removal of a concerning mole on the back with frankincense, a remedy that she found online.

A 43-year-old woman presented with a worrisome mole on the back. She had no personal history of skin cancer, but her father had a history of melanoma in situ in his 60s. The patient reported that she had the mole for years, but approximately 1 month prior to her visit she noticed that it began to bleed and crust, causing concern for melanoma. She read online that the lesion could be removed with topical application of the essential oil frankincense; she applied it directly to the lesion on the back. Within hours she developed a burn where it was applied with associated blistering.

Clinically, the lesion appeared as a darkly pigmented, well-circumscribed papule with hemorrhagic crust overlying a well-demarcated pink plaque (Figure 1). Dermatoscopically, the lesion lacked a pigment network and demonstrated
2 distinct pink papules with peripheral telangiectasia and a pink background with white streaks (Figure 2). A shave biopsy of the lesion demonstrated a nodular basal cell carcinoma extending to the base and margin.

Frankincense is the common name given to oleogum-resins of Boswellia species. ${ }^{1}$ It has been studied extensively for anti-inflammatory and antitumoral properties. It has been demonstrated that high concentrations of its active component, boswellic acid, can have a cytotoxic or cytostatic effect on certain malignant cell lines, such as melanoma, in vitro. ${ }^{2,3}$ It also has been shown to be antitumoral in mouse models. ${ }^{4}$ There are limited in vivo studies in the literature assessing the effects of boswellic acid or frankincense on cutaneous melanocytic lesions or other cutaneous malignancies, such as basal cell carcinoma.

A Google search of home remedy mole removal yielded more than 1,000,000 results. At the time of submission,

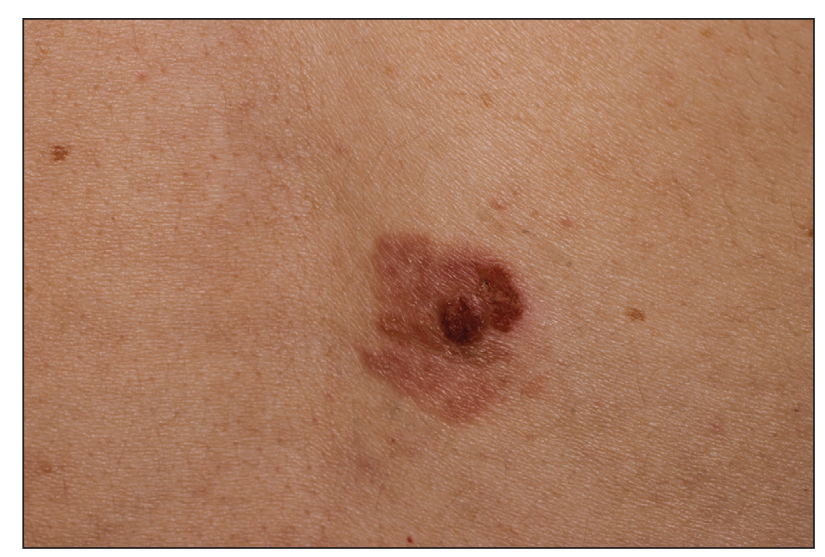

FIGURE 1. Darkly pigmented, well-circumscribed papule with hemorrhagic crust overlying a well-demarcated pink plaque.

\footnotetext{
From the Department of Dermatology and Cutaneous Biology, Thomas Jefferson University Hospital, Philadelphia, Pennsylvania. 


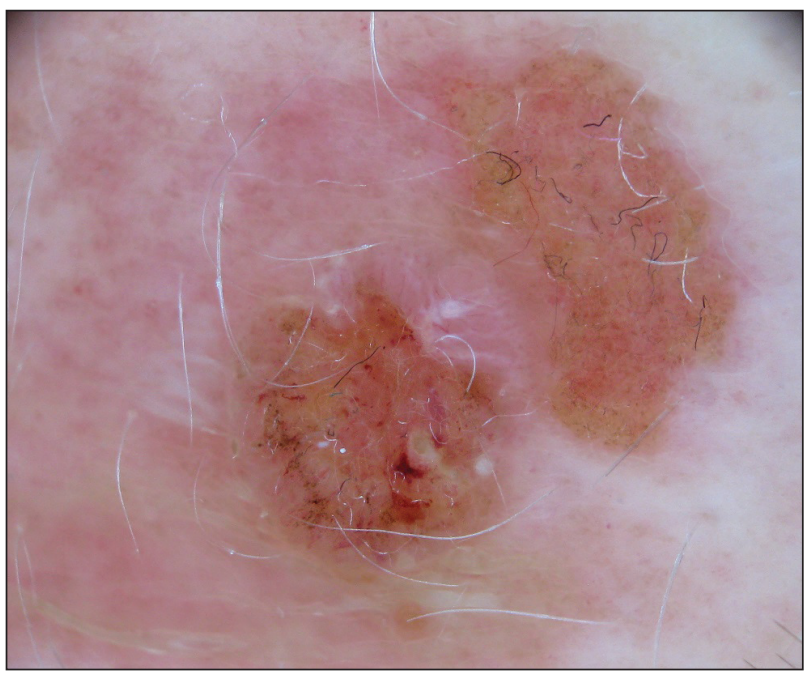

FIGURE 2. Dermatoscopic image demonstrating 2 distinct pink papules with peripheral telangiectasia on a pink background with white streaks.

the top 5 results all listed frankincense as a potential treatment along with garlic, iodine, castor oil, onion juice, pineapple juice, banana peels, honey, and aloe vera. None of the results cited evidence for their treatments. Although all recommended dilution of the frankincense prior to application, none warned of potential risks or side effects of its use.

Natural methods of home mole removal have long been sought after. Escharotics are most commonly utilized, including bloodroot (Sanguinaria canadensis), zinc chloride, Chelidonium majus, and Solanum sodomaeum. Many formulations are commercially available online, despite the fact that they can be mutilating and potentially dangerous when used without appropriate supervision. ${ }^{5}$ This case and an online search demonstrated that these agents are not only potentially harmful home remedies but also are currently falsely advertised as effective therapeutic management for melanocytic nevi.

Approximately 6 million individuals in the United States search the internet for health information daily, and as many as $41 \%$ of those do so to learn about alternative medicine. ${ }^{5,6}$ Although information gleaned from search engines can be useful, it is unregulated and often can be inaccurate. Clinicians generally are unaware of the erroneous material presented online and, therefore, cannot appropriately combat patient misinformation. Our case demonstrates the need to maintain an awareness of common online fallacies to better answer patient questions and guide them to more accurate sources of dermatologic information and appropriate treatment.

\section{REFERENCES}

1. Du Z, Liu Z, Ning Z, et al. Prospects of boswellic acids as potential pharmaceutics. Planta Med. 2015;81:259-271.

2. Eichhorn T, Greten HJ, Efferth T. Molecular determinants of the response of tumor cells to boswellic acids. Pharmaceuticals (Basel). 2011;4:1171-1182.

3. Zhao W, Entschladen F, Liu H, et al. Boswellic acid acetate induces differentiation and apoptosis in highly metastatic melanoma and fibrosarcoma cell. Cancer Detect Prev. 2003;27:67-75.

4. Huang MT, Badmaev V, Ding Y, et al. Anti-tumor and anticarcinogenic activities of triterpenoid, beta-boswellic acid. Biofactors. 2000;13:225-230

5. Adler BL, Friedman AJ. Safety \& efficacy of agents used for home mole removal and skin cancer treatment in the internet age, and analysis of cases. J Drugs Dermatol. 2013;12:1058-1063.

6. Kanthawala S, Vermeesch A, Given B, et al. Answers to health questions: internet search results versus online health community responses. J Med Internet Res. 2016;18:E95. 